\title{
Auslegung einer Einzelpol-Synchron- Reluktanzmaschine hinsichtlich sensorlosen Betriebs eines industriellen Antriebs
}

\author{
M. Hofer OVE, M. Nikowitz, M. Schrödl OVE
}

\begin{abstract}
In dieser Arbeit wird die Auslegung einer Synchron-Reluktanzmaschine mit ausgeprägten Polen für eine industrielle Applikation vorgestellt. Dieser Maschinentyp besticht durch seinen einfachen und kostengünstigen Aufbau ohne Permanentmagnete und Wicklungen im Rotor und der zusätzlichen Möglichkeit, geberlose Regelungsverfahren anwenden zu können. Die Auslegungskriterien werden erläutert und die Maschine auf Basis eines gegebenen Stators untersucht. Ein niedriger Oberschwingungsgehalt der Statorspannung sowie eine geringe Welligkeit des Drehmoments sind wesentliche Ziele beim Entwurf. Durch Variation von Geometrieparametern wird ein optimaler Rotorentwurf gefunden. Abschließend werden mögliche Betriebsstrategien der Maschine im Feldschwächbetrieb analysiert und dadurch die Einsatzmöglichkeit für diese Anwendung bestätigt.
\end{abstract}

Schlüsselwörter: Synchronmaschine; Reluktanzmaschine; elektrischer Antrieb; sensorlose Regelung

\section{Design of a salient-pole synchronous reluctance machine for an industrial drive considering sensorless operation.}

In this paper the design of a salient pole synchronous reluctance maschine for an industrial drive is discussed. This machine type shows advantages by the lack of permanentmagnets or windings in the rotor and the capability of sensorless rotor position detection, additionally. The design criteria for a given induction machine stator are presented. Low voltage harmonics and a low torque ripple are key requirements. By parameter variation an optimized rotor geometry is found. Finally, possible operation strategies in the flux weakening range are analyzed to confirm the machine design for this application.

Keywords: synchronous machine; electrical drive; sensorless control; reluctance machine

Eingegangen am 1. Dezember 2017, angenommen am 6. Februar 2018, online publiziert am 27. März 2018

(c) The Author(s) 2018. Dieser Artikel ist auf Springerlink.com mit Open Access verfügbar

\section{Einleitung}

Synchron-Reluktanzmaschinen (SynRM) haben in den letzten Jahren an Popularität gewonnen. Einerseits ist der Maschinentyp durch einen einfachen Rotoraufbau ohne Permanentmagnete und Rotorwicklungen gekennzeichnet und andererseits können damit IECWirkungsgradanforderungen der Klasse IE4 oder sogar IE5 [1] erreicht werden. Der alternative Einsatz von SynRM anstatt Asynchronmotoren (ASM) wurde in diversen Arbeiten [2-4] untersucht. Für diese Anwendung wurde bereits in einer vorgehenden Arbeit [5] ein SynRM-Rotor mit Flussbarrieren entworfen (Nenndaten in Tab. 1). Der Wirkungsgradvorteil dieser Maschine wurde durch eine deutlich geringere Erwärmung bei gleichem Anwendungsprofil bestätigt. Die Anforderungen aus der industriellen Anwendung an die elektrische Maschine können im Folgenden zusammengefasst werden:

- Die Übernahme des Stators einer Norm-Asynchronmaschine (zumindest die Statorgeometrie und die Wicklungsanordnung) der Baugröße 63.

- Ein Drehmoment von 0,6 N m bei 1,5 $A_{\text {eff }}$ Phasenstrom bis zumindest einer Drehzahl von $800 \mathrm{~min}^{-1}$

- Ein weiter Konstantleistungsbereich von $50 \mathrm{~W}$ bis zu einer Drehzahl von $6000 \mathrm{~min}^{-1}$ im Feldschwächbetrieb bei $162 V_{\text {eff }}$ Außenleiterspannung.

- Ein niedriger Drehmomentrippel und ein geringer Oberschwingungsgehalt der Statorspannung für eine gute Regelbarkeit und geringe Schwingungsneigung des Antriebs.
Tab. 1. Nenndaten der Synchron-Reluktanzmaschine

\begin{tabular}{ll}
\hline Nennleistung & $185 \mathrm{~W}$ \\
Nennmoment & $0,6 \mathrm{~N} \mathrm{~m}$ \\
Nenndrehzahl & $3000 \mathrm{~min}^{-1}$ \\
Nennstrom & $1,5 \mathrm{~A}_{\text {eff }}$ \\
Nennspannung & $162 \mathrm{~V}_{\text {eff, }} \mathrm{Y}$ \\
\hline
\end{tabular}

Mit der Verwendung einer SynRM anstatt einer ASM kann bereits eine Kostensenkung bei Material- und Fertigungskosten erreicht werden. Eine Kombination der SynRM mit einer sensorlosen Positionserfassung spart weiters die Gebereinheit und entsprechende Schnittstellen im Antriebssystem ein. Sensorlose Verfahren werden in einigen Anwendungen bereits industriell eingesetzt. Für SynRM wurde das sogenannte INFORM-Verfahren in [6] vorgestellt und auch in [7] erfolgreich angewendet. Auch für diese Anwendung wurde eine sensorlose Regelung der Flussbarrieren-SynRM einschließlich der Feldschwächung bereits in [5] untersucht. Aus Erfahrungen mit die-

Hofer, Matthias, Institut für Energiesysteme und Elektrische Antriebe, Technische Universität Wien, Gußhausstraße 25, 1040 Wien, Österreich

(E-Mail: matthias.hofer@tuwien.ac.at); Nikowitz, Mario, Institut für Energiesysteme und Elektrische Antriebe, Technische Universität Wien, Gußhausstraße 25, 1040 Wien, Österreich (E-Mail: mario.nikowitz@tuwien.ac.at); Schrödl, Manfred, Institut für Energiesysteme und Elektrische Antriebe, Technische Universität Wien, Gußhausstraße 25 1040 Wien, Österreich (E-Mail: manfred.schroedl@tuwien.ac.at) 
ser Maschine und eines magnetgelagerten sensorlosen Antriebs mit einer Einzelpol-SynRM [7] können zwei wesentliche Anforderungen für eine gute Eignung der SynRM zur Verwendung von sensorlosen Positionsbestimmungsverfahren abgeleitet werden:

- Eine hohe Achsigkeit durch ein großes Induktivitätsverhältnis $L_{d} / L_{q}$

- Möglichst konstante Induktivitätswerte der Längs- und Querinduktivität $L_{d}$ und $L_{q}$, welche unabhängig vom Betriebspunkt sind.

Ausgehend von diesen Gesamtanforderungen wird in dieser Arbeit eine SynRM mit ausgeprägten Polen entworfen, weil bei diesem Maschinentyp die Geometrie hauptverantortlich für die Induktivitäten sind und eine geringe Arbeitspunktabhängigkeit erwartet wird.

\subsection{Mathematische Beschreibung der SynRM}

Mit einer Beschreibung der SynRM im rotorfesten dq-Koordinatensystem kann die Achsigkeit der Maschine entsprechend berücksichtigt werden. Die komplexe Statorspannung $\underline{U}_{S}=U_{S, d}+j U_{S, q}$ kann durch die verkoppelten Differentialgleichungen

$$
\begin{aligned}
& U_{S, d}=R_{S} I_{S, d}+L_{d} \frac{d I_{S, d}}{d t}-\Omega_{e} L_{q} I_{S, q} \\
& U_{S, q}=R_{S} I_{S, q}+L_{q} \frac{d I_{S, q}}{d t}+\Omega_{e} L_{d} I_{S, d}
\end{aligned}
$$

beschrieben werden, wobei $R_{S}$ der Statorwiderstand, $\Omega_{e}$ die elektrische Kreisfrequenz, $L_{d}$ die Längs- und $L_{q}$ die Querinduktivität und $\underline{I}_{S}=I_{S, d}+j l_{S, q}$ der komplexe Statorstrom ist. Es sei erwähnt, dass im Allgemeinen die Maschineninduktivitäten nicht konstant und abhänging vom Arbeitspunkt sind, also mit $L_{d}\left(I_{S, d}, I_{S, q}\right)$ und $L_{q}\left(I_{S, d}, I_{S, q}\right)$ Funktionen der Stromkomponenten sind. Das Drehmoment $M$ ist abhängig von der Differenz der Induktivitäten und den Stromkompenenten in Längs- und Querrichtung

$$
M=\frac{3}{2} p\left(L_{d}-L_{q}\right) I_{S, d} I_{S, q}
$$

Ist eine der beiden Stromkomponenten Null wird kein Drehmoment erzeugt. Weiters ist bei einer geringen Achsigkeit $\left(L_{d}-L_{q}\right)$ die Drehmomentausbeute gering. Da speziell bei Flussbarrieren-SynRM die Induktivitäten über einen weiten Bereich variieren, resultiert daraus die äußerst geringe Drehmomententwicklung bei geringem Strom (Teillastbetrieb), solange die Rotorstege nicht gesättigt sind.

\subsection{Rotor mit Flussbarrieren}

Die SynRM mit Flussbarrieren im Rotor basieren üblicherweise auf dem Konzept durch die Anordnung von Bohrungen oder Schlitzen innerhalb der zylindrischen Rotorgeometrie eine Achsigkeit des Rotors zu erzeugen. Für diese Anwendung wurde bereits ein vierpoliger Rotor mit drei Flussbarrieren entworfen und aufgebaut (siehe [5]). Die gemessene stationäre Drehmoment/Drehzahl-Kennlinie in zwei verschiedenen Betriebsarten MTPA und MTPV (engl. Maximum Torque per Ampere, Maximum Torque per Volt) sowie die Drehmomentanforderung ist in Abb. 1 dargestellt. Dabei wurde der Stator der ASM vollständig (Windungszahl, $\Delta$-Schaltung) übernommen und die geforderten Drehmomente konnten erreicht werden. In dieser vorgehenden Arbeit wurde festgestellt, dass für den sensorlosen Betrieb dieser Maschine bei kleinen Drehzahlen inkl. Stillstand eine Mindestachsigkeit gefordert wird und diese nur durch eine permanente Bestromung mit einem Mindeststrom $I_{S, q, \text { min }}$ sichergestellt werden kann. Das bedeutet einen schlechteren Wirkungsgrad im Teillastbetrieb gegenüber einer Regelung mit Drehgeber und ist mitunter ein Grund für die nähere Untersuchung eines Einzelpol-Rotors.

Rotoren mit Flussbarrieren können auch eine nicht-zylindrische Außenkontur haben. Beispielsweise werden in [8] unterschiedliche

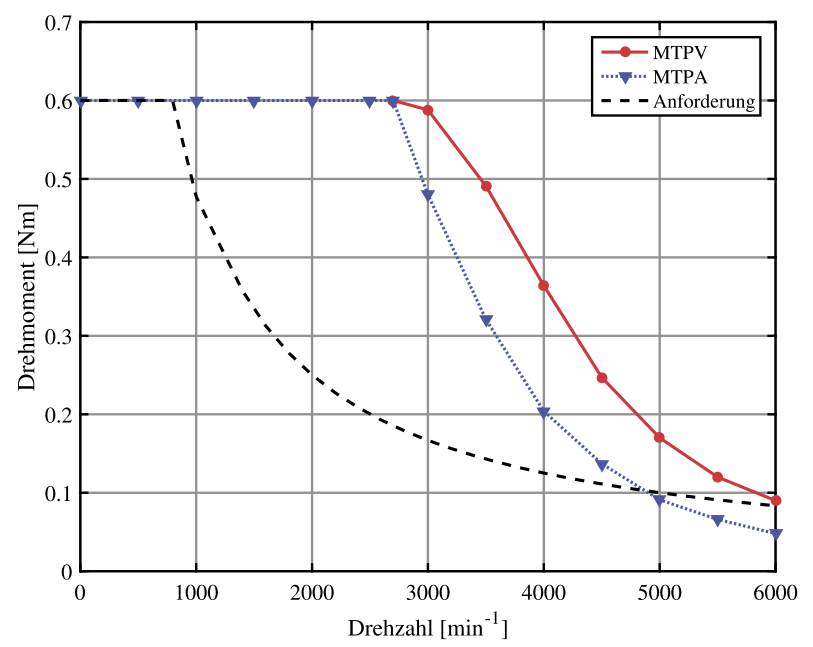

Abb. 1. Gemessene Drehmoment/Drehzahl-Kennlinie des Rotors mit Flussbarrieren in der MTPA- und MTPV-Betriebsart und Vergleich mit der Drehmoment-Anforderung der Anwendung

Rotorvarianten mit und ohne sogenannten „cut-offs" bzw. "cutouts" untersucht. Durch Vergrößerung der äußersten Flussbarriere nach außen hin bis zum Rotordurchmesser entsteht eine Ausnehmung in der q-Richtung an der Rotoroberfläche. Je nach Abmessungen und Anzahl der Flussbarrieren kann so eine Mischform zwischen Flussbarrieren-Rotor und Rotor mit ausgeprägten Polen dargestellt werden. In [8] wird dadurch ein etwas höheres Induktivitätsverhältnis $L_{d} / L_{q}$ erreicht, wodurch auch das maximale mittlere Drehmoment und der Wirkleistungsfaktor leicht ansteigen. Diese Ausnehmungen führen aber auch zu einem Anstieg des Drehmomentrippels. Aufgrund des Einflusses auf die Induktivitäten kann dadurch

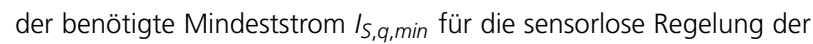
Flussbarrieren-SynRM je nach Ausführung der Rotor-Mischform verringert oder ev. auch ganz vermieden werden. Dieser Rotortyp ist daher ebenfalls für einen sensorlosen Betrieb denkbar, jedoch ist die Rotorgeometrie komplexer, wodurch höhere Fertigungskosten als beim Einzelpolrotor erwartet werden.

\subsection{Rotor mit ausgeprägten Polen}

Beim SynRM-Rotor mit Einzelpolen bzw. ausgeprägten Polen kommt die Achsigkeit alleine durch einen variablen Luftspalt am Umfang des Rotors zustande. Die Maschinenparameter sind daher weitgehend unabhängig vom Lastpunkt der Maschine. Durch die Vermeidung von internen Ausnehmungen im Rotor kommt es auch nicht zu lokal auftretenden hohen mechanischen Spannungen bei hohen Drehzahlen. Daher erlaubt der Einzelpol-Rotor den Betrieb bei höheren Drehzahlen als ein Flussbarrieren-Rotor gleichen Durchmessers. Der Prinzipquerschnitt eines vierpoligen Einzelpolrotors mit den wesentlichen Parametern Polbreite $b_{P}$, Polradius $r_{P}$ und Polwinkel $\beta_{P}$ ist in Abb. 2 gezeigt. Ein derartiger Rotor wurde für eine SynRM in $\Delta$-Schaltung bereits analysiert [9]. Die Analyse zeigt, dass hier eine hohe dritte Spannungskomponente auftritt und zu Problemen mit Kreisströmen führen kann. Eine Abhilfe bietet lediglich die Verwendung einer Zweischichtwicklung mit 5/6- oder 4/6-Sehung. In dieser Anwendung ist aber der Beibehalt der Werkzeuge und der Fertigungsschritte in der Statorfertigung gewünscht. Daher beruht die folgende Analyse auf einer Einschichtwicklung $(q=2)$ bei 24 Statornuten mit einer adaptierten Statorwindungszahl $N=74$ in Y-Schaltung. 


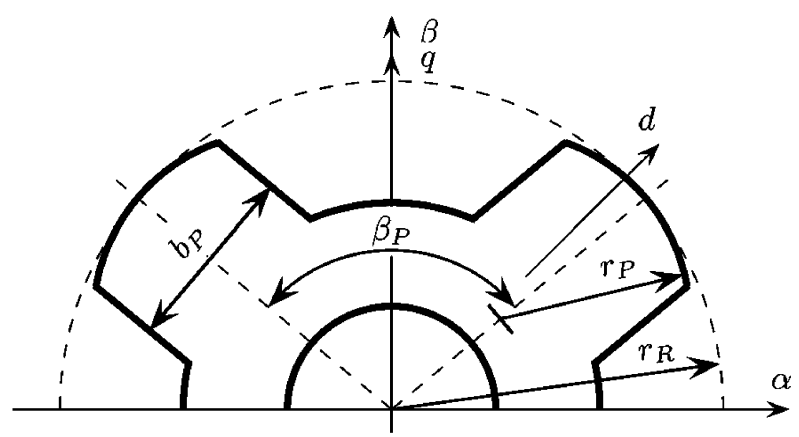

Abb. 2. Rotor mit ausgeprägten Polen $(2 p=4)$ und wesentliche Geometrieparameter

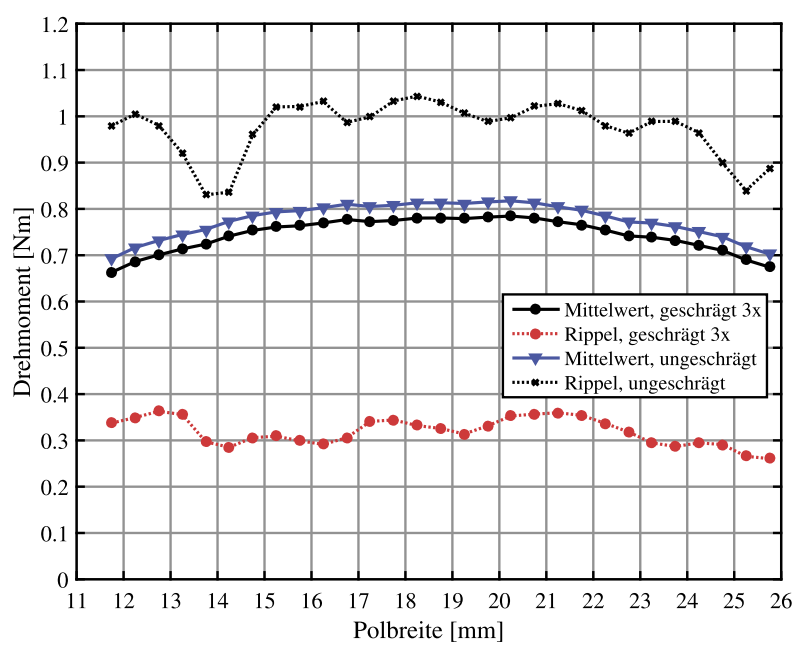

Abb. 3. Mittelwert und Rippel des Drehmoments in Abhängigkeit der Polbreite. Vergleich eines ungeschrägten und diskret geschrägten Rotors mit 3 Segmenten

\section{Maschinenentwurf}

In diesem Abschnitt wird der Maschinenentwurf der EinzelpolSynRM mit entsprechendem Parametereinfluss des Rotors diskutiert. Die Statorgeometrie und die Statorwicklung bleiben unverändert. Die Analyse erfolgt mittels zweidimensionaler Finite Elemente Methode bei sinusförmiger Stromeinprägung. Die diskrete Schrägung des Rotors wird durch Berechnung von "Teilmaschinen" und Superposition durchgeführt. Eine Berücksichtigung der Eisen- und Reibungsverluste erfolgt in diesem Entwurfsstadium noch nicht.

\subsection{Rotorpolbreite und diskrete Schrägung}

Ein wesentlicher Parameter des Rotors ist die Polbreite $b_{p}$. Die Variation (Abb. 3) bei zylindrischer Rotorform $\left(r_{P}=r_{R}\right)$ zeigt, dass in einem Bereich $16 \mathrm{~mm}$ bis $21 \mathrm{~mm}$ der Polbreite ein Plateau des Drehmoments liegt. Ebenfalls wurde eine diskrete Schrägung des Rotors um eine Statorzahnteilung $\left(15^{\circ}\right)$ mit 3 Segmenten untersucht, welche eine signifikante Reduktion des Drehmomentenrippels bringt. Der Rippel ist im ungeschrägten Fall etwa $1 \mathrm{Nm}$ und wird bereits durch Schrägung mit 3 Segmenten um etwa Faktor 3 auf etwa $0,3 \mathrm{~N} \mathrm{~m}$ reduziert. Weiters ist das harmonische Verhalten der AuBenleiterspannung in Abb. 4 ersichtlich. Die Fourier-Analyse zeigt ungerade Ordnungszahlen der Spannungsharmonischen (siehe auch Abb. 6). Die Spannungen $U_{5}, U_{7}, U_{11}$ und $U_{13}$ sind dabei dominant und It. Abb. 4 je nach Polbreite $b_{P}$ zusammen nahezu unabhängig

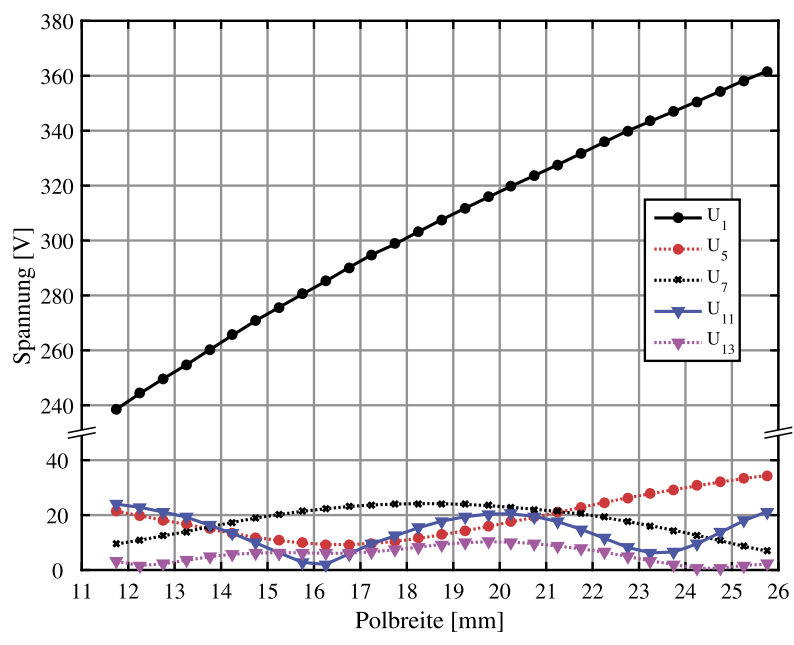

Abb. 4. Harmonische der Außenleiterspannung bei Nenndrehzahl und Nennstrom in Abhängigkeit der Polbreite des diskret geschrägten Rotors mit 3 Segmenten

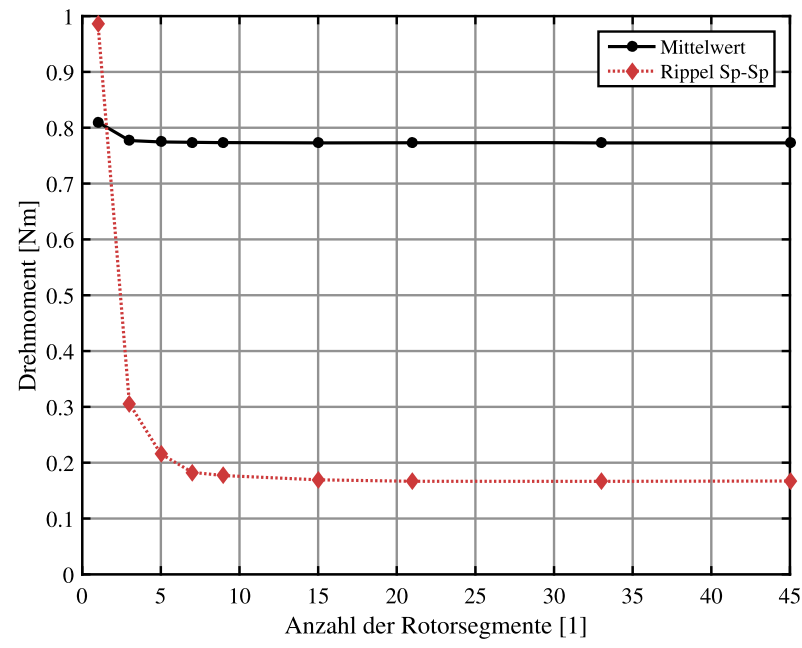

Abb. 5. Einfluss der Rotorelemente auf den Mittelwert und Rippel des Drehmoments bei Nenndrehzahl und Nennstrom

bei etwa 20 V Amplitude. Zur Erfüllung der Spannungsanforderung ist eine möglichst kleine Spannungsgrundschwingung $U_{1}$ bei einem Minimum der Oberschwingungen erwünscht. Diese wäre zwar bei $b_{P}=14 \mathrm{~mm}$ gegeben, was aber zu weniger Drehmoment führen würde, daher wurde $b_{p}=16,75 \mathrm{~mm}$ gewählt.

Die Rotorschrägung bringt hinsichtlich Drehmomentenwelligkeit schon mit 3 Segmenten eine deutliche Verbesserung. Eine Erhöhung der Segmentanzahl mit dem Einfluss auf das Drehmoment ist in Abb. 5 dargestellt. Während der Mittelwert bereits ab 3 Segmenten nicht mehr weiter sinkt, kann mit 9 Segmenten der gleiche Rippel erreicht werden wie bei kontinuierlichen Schrägung mit sehr großer Segmentanzahl. In der Fourier-Analyse Abb. 6 sieht man den großen Einfluss der Schrägung vor allem auf die höheren Harmonischen und eine deutliche Reduktion der Spannungen $U_{11}$ und $U_{13}$.

\subsection{Ungleiche Polabstände}

Üblicherweise ist die Geometrie und die Wicklungsanordnung von elektrischen Maschinen des Stators und Rotors symmetrisch und für 


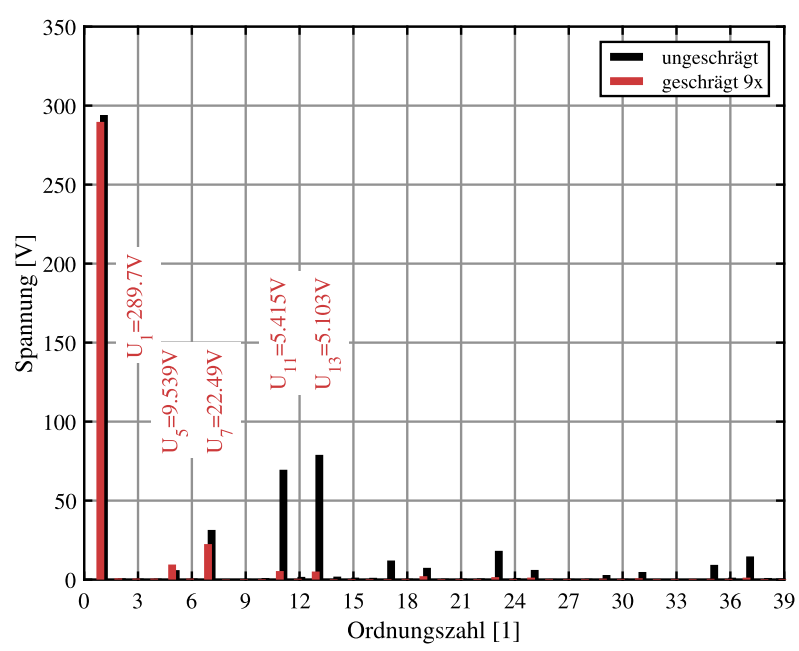

Abb. 6. Harmonische der Außenleiterspannung bei Nenndrehzahl und Nennstrom des diskret geschrägten Rotors mit 9 Segmenten

jeden Pol bzw. jedes Polpaar gleich ausgeführt. D. h. eine vierpolige Maschine $(2 p=4)$ besitzt vier gleiche Polformen in äquidistanten Polabständen von $\beta_{P}=90^{\circ}$. Die Verwendung von asymmetrischen Anordnungen, wie z. B. bei Flussbarrieren-SynRM [10], haben einen Einfluss auf die Spannungsharmonischen der Maschine und können als Entwurfsmaßnahmen zur Beeinflussung von Maschineneigenschaften herangezogen werden. Bei diesem Design einer EinzelpolSynRM wird der Abstand zwischen jeweils zwei Polen mit dem Winkel $\beta_{P}$ durch Verwendung eines Versatzwinkels $\Delta \beta$ zu

$$
\beta_{P, 1,2}=\frac{360^{\circ}}{2 p} \pm 2 \Delta \beta
$$

verändert. Damit ergeben sich für die vierpolige Maschine jeweils abwechselnd ein größerer Winkel $\beta_{P, 1}$ und ein kleinerer Winkel $\beta_{P, 2}$ zwischen zwei Polen. Positive oder negative Winkel $\Delta \beta$ führen jeweils zur gleichen Wirkung von zwei größen und zwei kleineren Polwinkeln. Im Allgemeinen wäre es natürlich möglich alle Winkel unterschiedlich groß zu wählen um ev. durch die weiteren Freiheitsgrade eine zusätzliche Verbesserung zu erreichen. Lt. Abb. 2 liegt dann die magnetische $d$-Achse nicht mehr direkt auf einer Polachse, sondern ist um den Winkel $\pm \Delta \beta$ verschoben. Die magnetische Querachse liegt weiterhin auf einer Symmetrieachse der Rotorgeometrie. Mit steigendem Polwinkel $\Delta \beta$ nimmt das Drehmoment (Abb. 7) und die Spannungsgrundschwingung $U_{1}$ (Abb. 8) ab. Es zeigt sich ein minimaler Drehmomentrippel bei einem Polwinkel $\Delta \beta=8^{\circ}$. Die Spannungsoberschwinung, vor allem die Spannung $U_{7}$ ist ebenfalls deutlich abgeschwächt. Dieser Polwinkel $\Delta \beta=8^{\circ}$ stellt einen guten Kompromiss zwischen Spannungsharmonischen und Drehmomentwelligkeit dar.

\subsection{Polform des Rotors}

Eine spezielle Ausformung der Rotorpolschuhe stellt ebenfalls eine Möglichkeit zur Beeinflussung der Harmonischen dar. Oftmals werden Polschuhe bei Schenkelpolmaschinen oder Permanentmagnetsynchronmaschine (PMSM) mit einer inversen Cosinusfuktion (z. B. $[11,12])$ entworfen. Ein kreisförmiger Pol mit einem Polradius $r_{P}$, welcher kleiner als der Rotorradius $r_{R}=27,8 \mathrm{~mm}$ ist, stellt eine gute Näherung dar [12] und ist konstruktiv entsprechend einfacher. Ausgehend von einem zylindrischen Rotor führt eine Verringerung des Polradius $r_{p}$ zur Reduktion der Spannungsgrundschwingung (Abb. 9) und zum Absinken des mittleren Drehmoments (Abb. 10). Das Rippelmoment sinkt nur leicht ab. Die größte

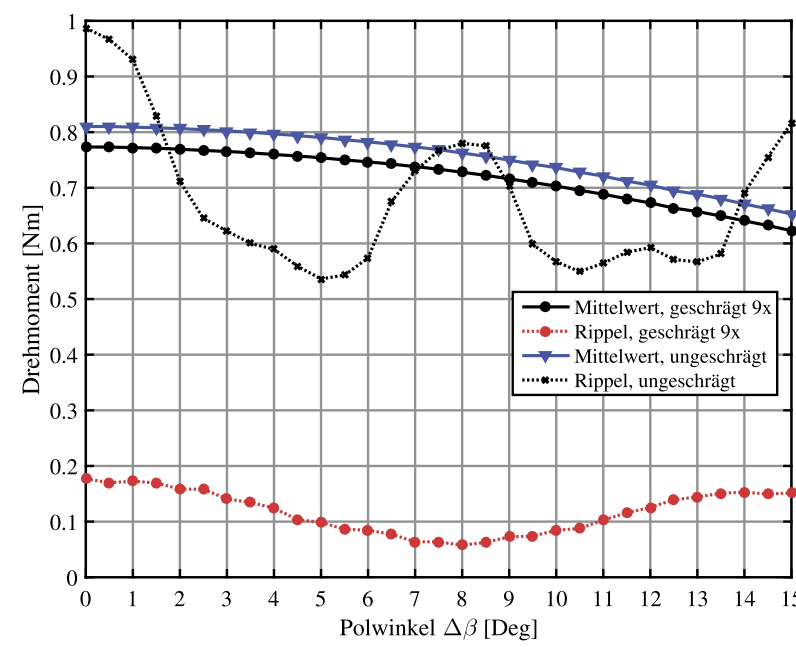

Abb. 7. Mittelwert und Rippel des Drehmoments des ungeschrägten und diskret geschrägten Rotors mit 9 Segmenten in Abhängigkeit des Polwinkels $\Delta \beta$

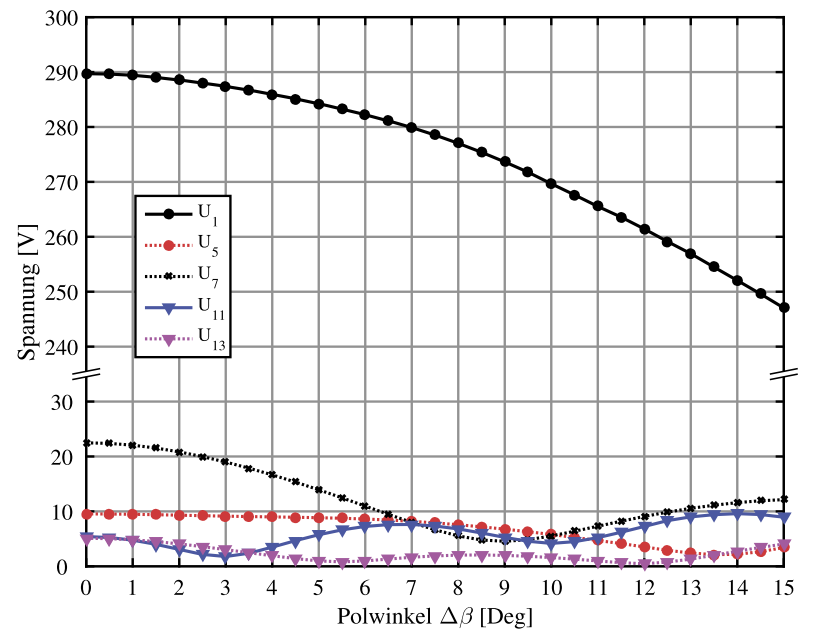

Abb. 8. Harmonische der Außenleiterspannung bei Nenndrehzahl und Nennstrom des diskret geschrägten Rotors mit 9 Segmenten in Abhängigkeit des Polwinkels $\Delta \beta$

Verbesserung des Drehmomentrippels hat die Rotorschrägung gebracht. Die Amplituden der Spannung mit den Ordnungszahlen 5, 7, 11 und 13 variieren nur marginal und steigen bei kleineren Radien sogar wieder leicht. Es zeigt sich also ein geringer Einfluss der Rotorpolform, dennoch wird für die Maschine ein um $2 \mathrm{~mm}$ kleinerer Polradius $r_{P}=25,8 \mathrm{~mm}$ verwendet. Die Statorspannungsgrundwelle beträgt $U_{1}=264,3 \mathrm{~V}$ bei Nenndrehzahl und das Drehmoment $M=0,685 \mathrm{Nm}$ liegt über der Anforderung. Um die Anforderungen des Antriebs zu erfüllen wird schließlich eine Verringerung der Windungszahl von $N=74$ auf $N=70$ vorgenommen. Folglich sinken bei Nennstrom die Drehmomente und die Spannungen.

\section{Betriebsverhalten der Einzelpol-SynRM}

Nach dem Entwurf der Maschine wird in diesem Abschnitt das Betriebsverhalten im Grunddrehzahl- und Feldschwächbetrieb untersucht. Zunächst sind die maximale Drehmomentausbeute und die Induktivitätswerte der Maschine von Interesse. Nachfolgend wird die Feldschwächung im MTPA- und MTPV-Betrieb erläutert. 


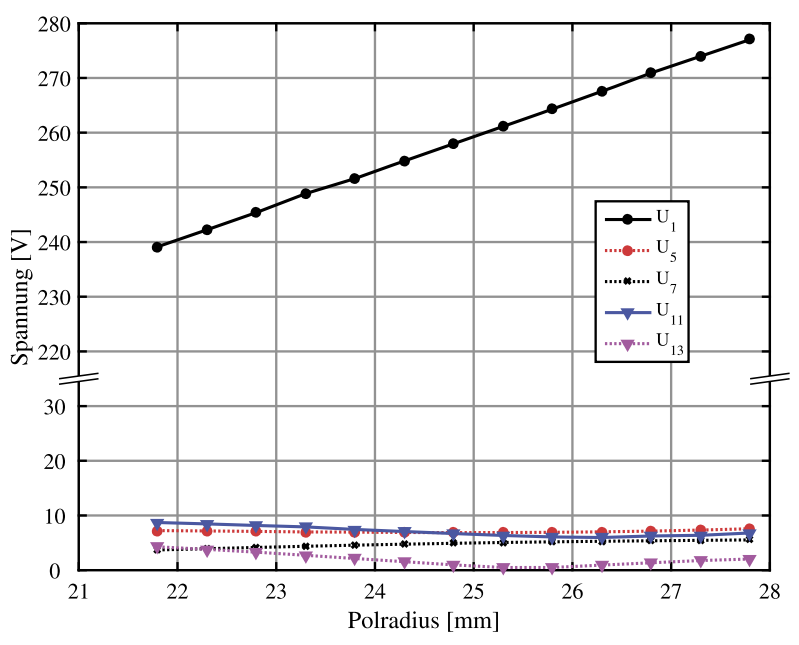

Abb. 9. Harmonische der Außenleiterspannung bei Nenndrehzahl und Nennstrom des diskret geschrägten Rotors mit 9 Segmenten, Polbreite $b_{P}=16,75 \mathrm{~mm}$, Polwinkel $\Delta \beta=8^{\circ}$ in Abhängigkeit des Polradius $r_{P}$

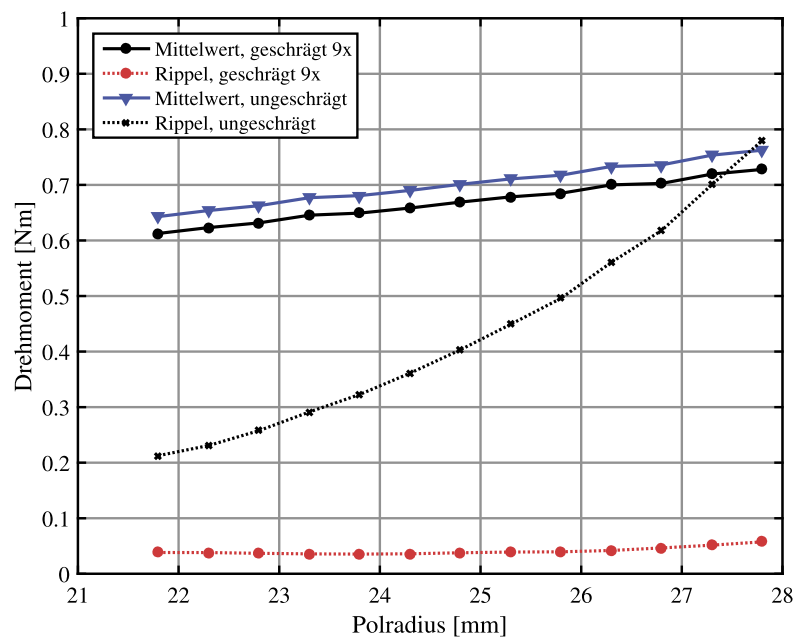

Abb. 10. Mittelwert und Rippel des Drehmoments des ungeschrägten und des diskret geschrägten Rotors mit 9 Segmenten, Polbreite $b_{P}=16,75 \mathrm{~mm}$ in Abhängigkeit des Polradius $r_{P}$

\subsection{Maximales Drehmoment pro Statorstrom}

Bei der Untersuchung It. Abb. 11 wird bei konstantem Strombetrag (Nennstrom und halber Nennstrom) der Stromwinkel $\arg \left\{I_{s, d q}\right\}$ variiert, um das Maximum des Drehmoments zu ermitteln (engl. Maximum Torque per Ampere, MTPA). Der Höchstwert liegt sowohl bei Nennenstrom und halben Nennstrom erwartungsgemäß bei $45^{\circ}$. Eine Verschiebung zu höheren Werten wie oft bei gesättigten Flussbarrieren-Maschinen ist hier aufgrund nahezu konstanter Induktivitäten nicht gegeben. Mit den optimalen Strömen $I_{S, d}=I_{S, q}=\left|I_{S}\right| / \sqrt{2}$ für diesen Maschinentyp im MTPA-Bereich erhält man somit den quadratischen Zusammenhang zwischen Drehmoment und Spitzenwert des Statorstroms zu

$$
M_{\text {MTPA }}=\frac{3}{4} p\left(L_{d}-L_{q}\right)\left|I_{S}\right|^{2}
$$

Der Rippel des Drehmoments ist dabei unabhängig vom Stromwinkel und ist ebenfalls wie das Drehmoment bei halbem Nennstrom

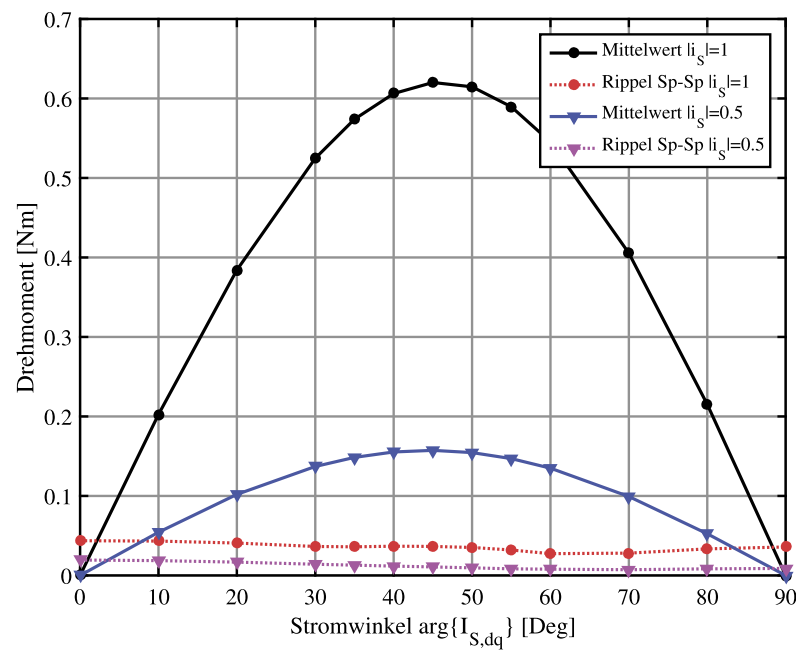

Abb. 11. Mittelwert und Rippel des Drehmoments des diskret geschrägten Rotors mit 9 Segmenten Polbreite $b_{P}=16,75 \mathrm{~mm}$, Polwinkel $\Delta \beta=8^{\circ}$, Polradius $r_{P}=25,8 \mathrm{~mm}$, Windungszahl $N=70$ bei Nennstrom und halben Nennstrom in Abhängigkeit des Stromwinkels $\arg \left\{\underline{I}_{s, d q}\right\}$

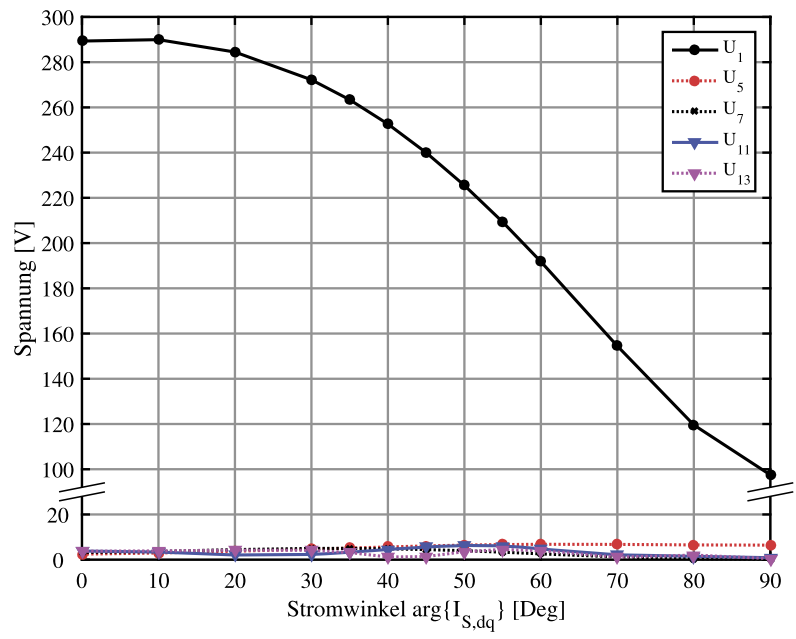

Abb. 12. Harmonische der Außenleiterspannung bei Nenndrehzahl und Nennstrom des diskret geschrägten Rotors mit 9 Segmenten, Polbreite $b_{P}=16,75 \mathrm{~mm}$, Polwinkel $\Delta \beta=8^{\circ}$, Polradius $r_{P}=25,8 \mathrm{~mm}$, Windungszahl $N=70$ bei Nennstrom in Abhängigkeit des Stromwinkels $\arg \left\{\underline{I}_{S, d q}\right\}$

etwa nur ein Viertel so groß. Das maximale Drehmoment liegt über der Anforderung von 0,6 N m. Aus der Analyse der Statorspannung (Abb. 12) ist ersichtlich, dass bei Nenndrehzahl $n_{N}=3000 \mathrm{~min}^{-1}$ bereits ein leicht feldschwächender Strom $\left(\arg \left\{I_{s, d q}\right\}=49^{\circ}\right)$ nötig ist, damit die Spannungsgrenze eingehalten wird. Die Harmonischen sind dabei nahezu unabhängig vom Arbeitspunkt und liegen im Bereich $<10 \mathrm{~V}$.

\subsection{Längs- und Querinduktivität}

Die Achsigkeit der Maschine wird durch die Induktivitäten charakterisiert. Die Analyse zeigt die klaren Vorteile des Rotors mit ausgeprägten Polen gegenüber Flussbarrieren-Typen. Die Längsinduktivität $L_{d} \approx 134 \mathrm{mH}$ ist nahezu und die Querinduktivität $L_{q}=38 \mathrm{mH}$ 


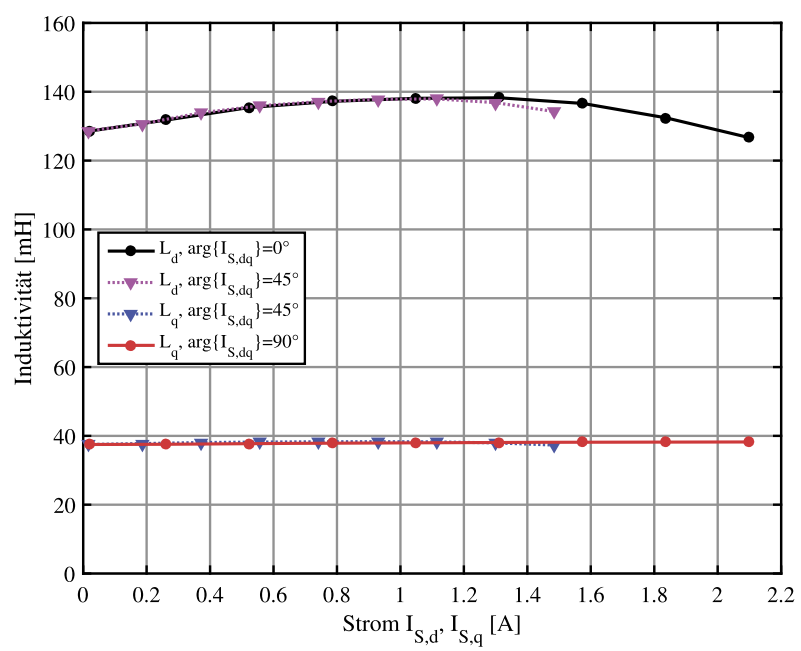

Abb. 13. Längs- und Querinduktivität $L_{d}$ und $L_{q}$ bei unterschiedlichen Stromwinkeln $\arg \left\{I_{S, d q}\right\}=0^{\circ}, 45^{\circ}, 90^{\circ}$

vollkommen unabhängig vom Arbeitspunkt der Maschine (Abb. 13). Eine vernachlässigbar kleine Gegeninduktivität $L_{d q}$ zufolge Sättigung gibt es daher nur bei großen Strömen. Diese Maschine hat ein mittleres und nahezu konstantes Verhältnis $L_{d} / L_{q}=3,52$. Diese Eigenschaft ist einerseits für die Verwendung einer sensorlosen Regelung äußerst vorteilhaft und ermöglicht andererseits die Verwendung einfacher linearer Maschinenmodelle It. Gl. (1) mit konstanten Koeffizienten für die Regelung. Im Allgemeinen werden speziell für die optimale Regelung der Maschine im Feldschwächbetrieb Kennfelder $L_{d}\left(I_{S, d}, I_{S, q}\right)$ und $L_{q}\left(I_{S, d}, I_{S, q}\right)$ der Induktivitäten verwendet, um Sättigung und ev. Querkopplung entsprechend zu berücksichtigen.

\subsection{Feldschwächung im MTPA-Betrieb}

Die MTPA-Betriebsart ist dadurch gekennzeichnet, dass der optimale Stromwinkel, in diesem Fall konstant $45^{\circ}$ verwendet wird $\left(I_{S, d}=I_{S, q}\right)$, siehe Abb. 11. Somit wird ein minimaler Strom für die Erzeugung des angeforderten Drehmoments verwendet was gleichbedeutend mit minimalen ohm'sche Verlusten ist. Diese Betriebsart wird üblicherweise im Grunddrehzahlbereich (Bereich I), solange die Spannungsgrenze nicht erreicht ist, verwendet und kann aber auch im Feldschwächbetrieb angewendet werden (Bereich II), siehe Abb. 14 und Abb. 15. Dabei bleibt der Stromwinkel konstant und der Strombetrag wird beim Erreichen der Spannungsgrenze bei $n=2850$ min $^{-1}$ reduziert. Genau an der Spannungsgrenze ist die Leistung maximal $(185 \mathrm{~W})$ und sinkt danach wieder bis auf $91 \mathrm{~W}$ bei $6000 \mathrm{~min}^{-1} \mathrm{ab}$.

\subsection{Feldschwächung im MTPV-Betrieb}

Abhängig von der Drehzahl der Maschine können nun drei Betriebsbereiche unterschieden werden (Abb. 16). Im Grunddrehzahlbereich (Bereich I) wird wieder der wirkungsgradoptimale MTPA-Betrieb verwendet. Ab der Spannungsgrenze bei $n=2850 \mathrm{~min}^{-1}$ wird in den MTPV-Betrieb übergegangen. Dabei wird das maximal erreichbare Drehmoment (bzw. Leistung) an der Spannungsgrenze ermittelt. Der Strombetrag wird dabei nicht reduziert, sondern vorerst weiterhin voll ausgenützt. Der Stromwinkel $\arg \left\{I_{s, d q}\right\}$ aber steigt von $45^{\circ}$ bis $73^{\circ}$ an (Bereich II). In diesem (näherungsweisen) Konstantleistungsbereich (engl. Constant Power Speed Range, CPSR) wird die q-Stromkomponente erhöht, während die $d$-Stromkomponente verringert wird (Abb. 17). Die Leistung der Maschine kann dadurch

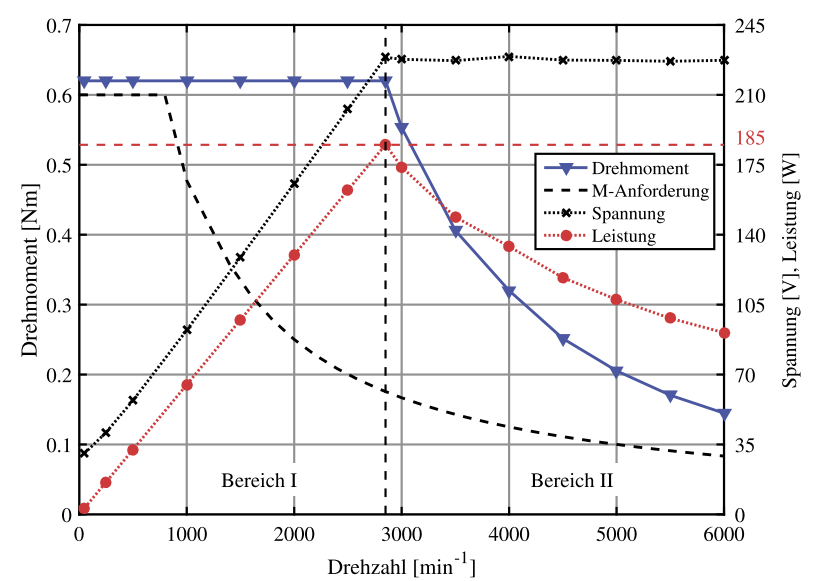

Abb. 14. Drehmoment, Außenleiterspannung $U_{1}$, Luftspaltleistung und Drehmoment-Anforderung in Abhängigkeit der Drehzahl im MTPA-Betrieb

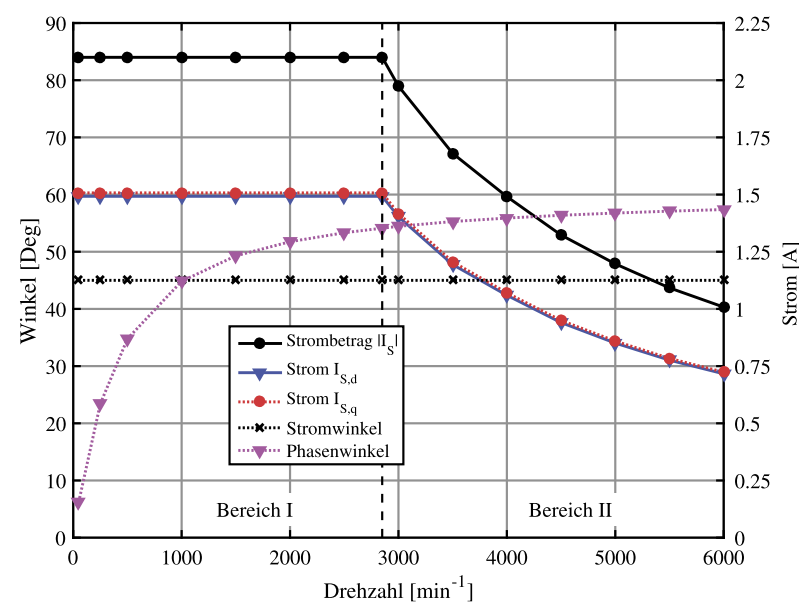

Abb. 15. Stromwinkel $\arg \left\{I_{S, d q}\right\}$ und Phasenwinkel $\varphi_{1}$, sowie Stromkomponenten $I_{S, d}$ und $I_{S, q}$ und Strombetrag $\left|I_{S}\right|$ in Abhängigkeit der Drehzahl im MTPA-Betrieb

noch weiter erhöht werden (>185 W) und ist an den Bereichsgrenzen I-II und II-III gleich groß (185 W). Im Bereich III wird dann sowohl der $\mathrm{d}$-als auch der q-Strom verringert und die Leistung sinkt mit steigender Drehzahl entsprechend ab.

Der Phasenwinkel $\varphi_{1}$ ist für Drehzahlen über $1500 \mathrm{~min}^{-1}$ etwa im Bereich $50^{\circ}$ bis $55^{\circ}$, was einem Wirkleistungsfaktor $\cos \varphi_{1} \approx 0,6$ entspricht. Dieser relativ geringe Wert ist typisch für SynRM oder auch ASM verglichen mit höheren Werten von PMSM. In der MTPVBetriebsart wird die Leistungsfähigkeit des SynRM-Antriebs im Feldschwächbetrieb gegenüber reinem MTPA-Betrieb deutlich erhöht. Weiters wird festgestellt, dass die Einzelpol-SynRM zwar bereits im MTPA-Betrieb die Drehmomentanforderungen an den Antrieb erfüllt und im MTPV-Betrieb bei $6000 \mathrm{~min}^{-1}$ sogar eine Luftspaltleistung von $158 \mathrm{~W}$, also etwa dreimal größer als die geforderte Leistung ist. Diese Analyse zeigt das hohe Potential der Einzelpolmaschine hinsichtlich Leistungsfähigkeit im Feldschwächbetrieb und auch der guten Eignung für eine sensorlose Regelung des Antriebs. 


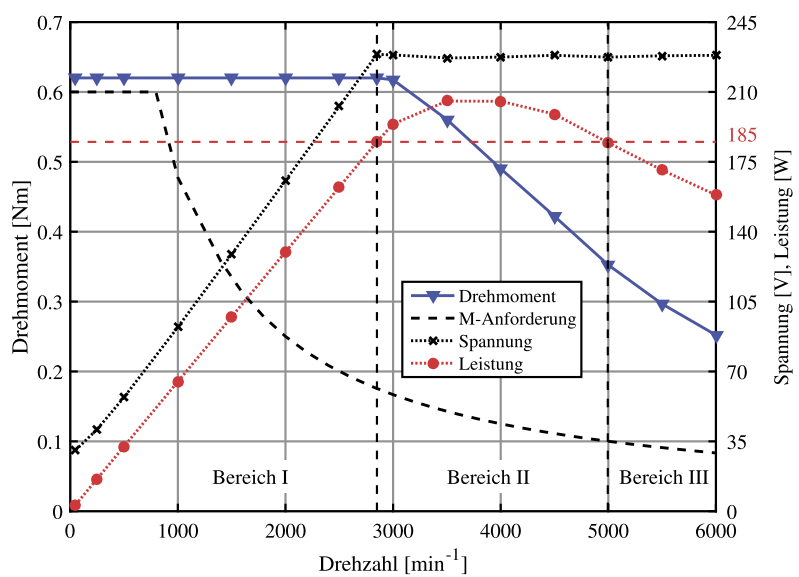

Abb. 16. Drehmoment, Außenleiterspannung $U_{1}$, Luftspaltleistung und Drehmoment-Anforderung in Abhängigkeit der Drehzahl im MTPV-Betrieb, Drehzahlbereiche I, II, III

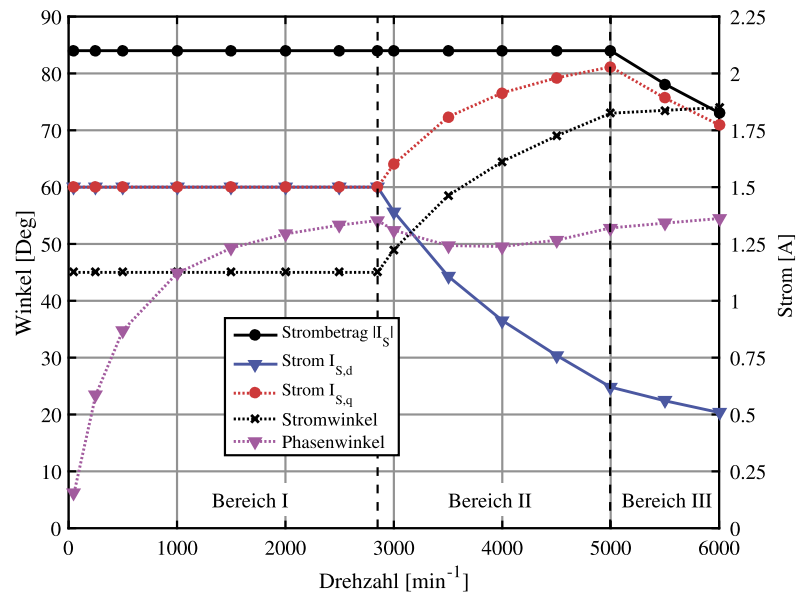

Abb. 17. Stromwinkel $\arg \left\{I_{S, d q}\right\}$ und Phasenwinkel $\varphi_{1}$, sowie Stromkomponenten $I_{S, d}$ und $I_{S, q}$ und Strombetrag $\left|I_{S}\right|$ in Abhängigkeit der Drehzahl im MTPV-Betrieb, Drehzahlbereiche I, II, III

\section{Zusammenfassung}

Die Untersuchung der Einzelpol-Synrchonreluktanzmaschine in dieser Arbeit zeigt vier wesentliche Vorteile gegenüber der Flussbarrieren-Reluktanzmaschine aufgrund eines hohen und konstanten Induktivitätsverhältnisses auf. Erstens, die hohe und konstante Achsigkeit ermöglicht eine sensorlose Positionsbestimmung unabhängig vom Laststrom auch im stromlosen Zustand. Zweitens, im Teillastbetrieb erzeugt die Einzelpol-Maschine größere Drehmomente bei gleichem Statorstrom, da kein Magnetisierungsbedarf zum Aussättigen der Stege wie beim Flussbarrieren-Rotor nötig ist. Drittens, im Feldschwächbetrieb an der Spannungsgrenze können höhere Drehmomente und Leistungen abgegeben werden. Viertens, die konstanten Maschinenparameter erlauben die Verwendung einer konventionellen feldorientierten Regelungsstruktur ohne Parameterkennfelder mit hoher Modellgenauigkeit. Wesentlich ist noch, dass der Rotor durch einfache und unkritische Fertigungsschritte kostengünstig erzeugt werden kann. Als nachteilig gezeigt hat sich der etwas höhere Aufwand beim Entwurf der Maschine und dass aufgrund der einfachen Geometrie nur wenige Parameter für eine Optimierung zur Verfügung stehen. Für die Anwendung in einem industriellen Antrieb konnte aber schließlich ein Rotor entworfen werden, welcher die Anforderungen des Antriebs selbst voll erfüllt und eine weitere Kosten- und Strukturvereinfachung mittels sensorloser Positionsbestimmung ermöglicht. Der entworfene Rotor wird derzeit als Prototyp gebaut und nach Fertigstellung hinsichtlich der Anforderungen mit einer sensorlosen Regelung verifiziert.

\section{Danksagung}

Open access funding provided by TU Wien (TUW).

Open Access This article is distributed under the terms of the Creative Commons Attribution 4.0 International License (http://creativecommons.org/ licenses/by/4.0/), which permits unrestricted use, distribution, and reproduction in any medium, provided you give appropriate credit to the original author(s) and the source, provide a link to the Creative Commons license, and indicate if changes were made.

\section{Literatur}

1. Dmitrievskii, V., Prakht, V., Kazakbaev, V., Oshurbekov, S., Sokolov, I. (2016): Developing ultra premium efficiency (IE5 class) magnet-free synchronous reluctance motor. In The 6th international electric drives production conference, EDPC.

2. Lipo Thomas, A. (1991): Synchronous reluctance machines - a viable alternative for AC drives? Electr. Mach. Power Syst., 19(6), 659-671.

3. Boglietti, A., Pastorelli, M. (2008): Induction and synchronous reluctance motors comparison. In The 34th annual conference of IEEE industrial electronics IECON, S. 20412044.

4. de Almeida, A., Ferreira, F., Baoming, G. (2014): Beyond induction motorstechnology trends to move up efficiency. IEEE Trans. Ind. Appl., 50(3), 2103-2114.

5. Hofer, M., Nikowitz, M., Schrödl, M. (2017): Application of a position sensorless control to a reluctance synchronous drive including flux weakening. In International exhibition and conference for power electronics, intelligent motion, Nürnberg, Deutschland: Renewable Energy and Energy Management, PCIM.

6. Schrödl, M., Weinmeier, P. (1994): Sensorless control of reluctance machine at arbitrary operation conditions including standstill. IEEE Trans. Power Electron., 9, 225-231.

7. Hofer, M., Spiessberger, R., Schrödl, M. (2015): Design and sensorless control of a reluctance synchronous machine for a magnetically levitated drive. In Proceedings of the 2015 IEEE international electric machines and drives conference, IEMDC.

8. Muteba, M., Twala, B., Nicolea, D. V. (2017): Based 3D finite element analysis of a synchronous reluctance motor with sinusoidal rotor shape. In Proceedings of the 2017 IEEE international electric machines and drives conference, IEMDC.

9. Hofer, M., Schrödl, M. (2017): Optimization of a synchronous reluctance machine for an industrial drive application regarding to sensorless control. In The 19th European conference on power electronics and applications. EPE'17 ECCE Europe.

10. Pina, A. J., Longya, X. (2015): Modeling of synchronous reluctance motors aided by permanent magnets with asymmetric rotor poles. In Proceedings of the 2015 IEEE international electric machines and drives conference, IEMDC.

11. Evans St, A. (2010): Salient pole shoe shapes of interior permanent magnet synchronous machines. In The XIX international conference on electrical machines, ICEM.

12. Richter, R. (1963): Elektrische Maschinen, 2. Band: Synchronmaschinen und Einankerumformer. Basel: Springer. ISBN 978-3-0348-4065-1. 


\section{Autoren}

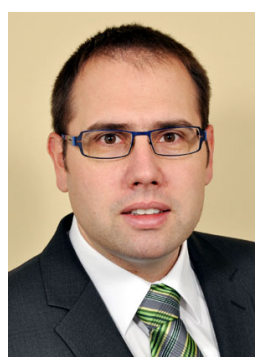

\section{Matthias Hofer}

Studium der Elektrotechnik an der Technischen Universität Wien, Österreich, Studienzweig Automatisierungs- und Regelungstechnik; Abschluss 2004. Universitäts- und Projektassistent am Institut für Elektrische Maschinen und Antriebe der TU Wien 2004 bis 2009, Projektleiter und Abteilungsleiter für die Berechnung elektrischer Maschinen bei Magna Electronics und Magna Powertrain 2009 bis 2013, 2013 Promotion zum Dr. techn., seit 2013 Universitätsassistent am Institut für Energiesysteme und Elektrische Antriebe an der TU Wien.

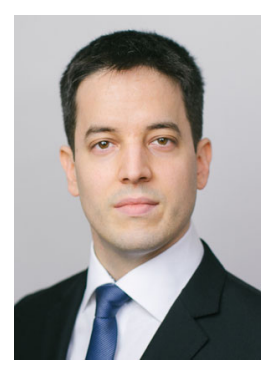

\section{Mario Nikowitz}

Studium der Elektrotechnik an der Technischen Universität Wien, Österreich, Studienzweig Automatisierungstechnik; Abschluss 2016. Seit 2016 Universitätsassistent am Institut für Energiesysteme und Elektrische Antriebe an der TU Wien.

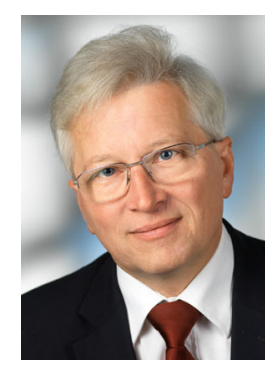

\section{Manfred Schrödl}

Studium der Elektrotechnik an der Technischen Universität Wien, Österreich, Studienzweig Industrielle Elektronik und Regelungstechnik; Abschluss 1982. Universitätsassistent am Institut für Elektrische Maschinen und Antriebe der TU Wien, 1987 Promotion zum Dr. techn., 1992 Habilitation für Elektrische Antriebe und Leistungselektronik in der Antriebstechnik. 1993 bis 1996 Leiter F\&E bei Elin Verkehrstechnik Wien, 1996 bis 1998 Bereichsleiter Zentrale Technik bei Flender ATB, Spielberg, Steiermark. Seit 1998 Ordentlicher Universitätsprofessor für Elektrische Antriebe und Maschinen sowie Vorstand des Instituts für Energiesysteme und Elektrische Antriebe an der TU Wien. 